\title{
The additional lead electrocardiogram in acute myocardial infarction
}

\author{
William J Brady, Francis Morris
}

In the evaluation of the patient with chest pain, the electrocardiogram (ECG) is an invaluable tool, assisting the physician in establishing certain diagnoses, selecting both appropriate therapies and inpatient disposition locations, and predicting risk of complication and death. The 12 lead ECG, however, is a less than perfect indicator of acute myocardial infraction (AMI), particularly when used early in the course of the acute ischaemic event. The sensitivity of a single 12 lead ECG for the diagnosis of AMI is relatively poor. For example, one report examined the initial ECG in a group of adult patients with chest pain, all of whom were subsequently proved to have had an AMI. ${ }^{1}$ Fewer than $50 \%$ of these patients demonstrated ST segment elevation on their initial ECG; the remaining patients manifested a variety of ischaemic changes, including ST segment depression and $\mathrm{T}$ wave inversion. In $30 \%$ of these patients the initial ECG was normal or showed non-specific changes only. This same study showed that ST segment depression was a poor indicator of AMI; fewer than $50 \%$ of those patients who presented with isolated precordial ST segment depression were subsequently shown to have had an AMI.

It has been suggested that the sensitivity of the 12 lead ECG may be improved if three additional body surface leads are employed in selected individuals. ${ }^{23}$ Acute posterior and right ventricular myocardial infarction are likely to be underdiagnosed, as the standard lead placement of the 12 lead ECG does not allow these areas to be assessed directly. ${ }^{4}$ Additional leads frequently used include leads V8 and V9 which image the posterior wall of the left ventricle and lead RV4 which reflects the status of the right ventricle. The standard ECG coupled with these additional leads constitute the 15 lead ECG, the most frequently employed extra lead in clinical practice. The use of the additional leads may not only confirm the presence of AMI but also provide a more accurate reflection of the true extent of myocardial damage. ${ }^{5}$ The following case reports demonstrate the use of the 15 lead ECG in clinical practice.

\section{Case reports}

\section{CASE 1}

A 79 year old women presented with retrosternal chest pain that radiated into her back of three hours' duration. The symptoms had started while she was travelling on a bus and had made her feel dizzy and sweaty. She was a heavy smoker with a history of exertional chest pain. She was diaphoretic and pale; the examination was otherwise unremarkable. The ECGs (fig 1) revealed a junctional rhythm with widespread ST depression in leads V1-V6. The electrocardiographic findings were felt to be compatible with either anterior ischaemia or isolated acute posterior wall infarction. Additional leads imaging the posterior wall (fig 2) were used which demonstrated ST segment elevation in leads V7-V9, confirming the presence of an acute posterior wall myocardial infarction. Cardiac enzymes were raised and this established the diagnosis of AMI.

CASE 2

A 62 year old man with a past history of hypertension developed epigastric pain at work. He

\section{Department of \\ Emergency Medicine, University of Virginia School of Medicine, Charlottesville, Virginia, USA W J Brady}

\section{Department of} Accident and Emergency Medicine, Northern General Hospital, Sheffield F Morris

Correspondence to: Dr William J Brady, Department of Emergency Medicine, Box 523-21, University of Virginia Health Sciences Centre,

Charlottesville, VA 22908,

USA (e-mail:

wb4z@hscmail.

mcc.virgina.edu).

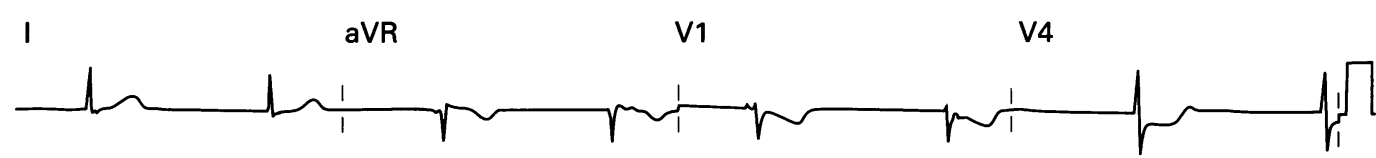

$\begin{array}{llll}\text { II } & \text { aVL } & \text { V2 } & \text { V5 }\end{array}$

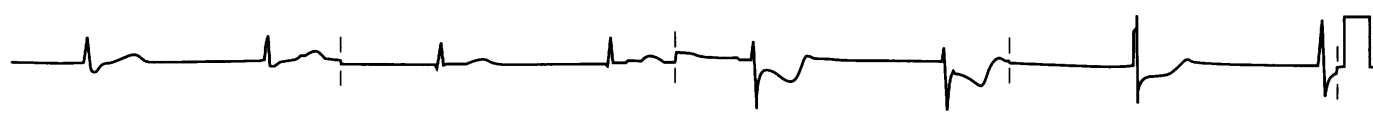

III aVF V3 V V

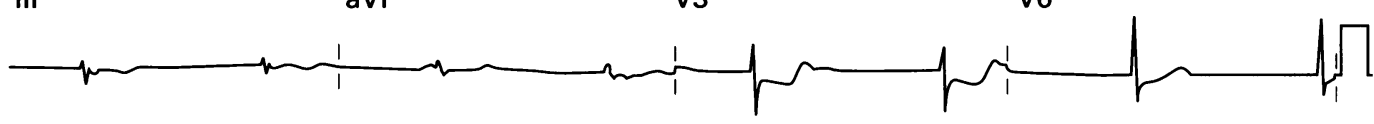

II

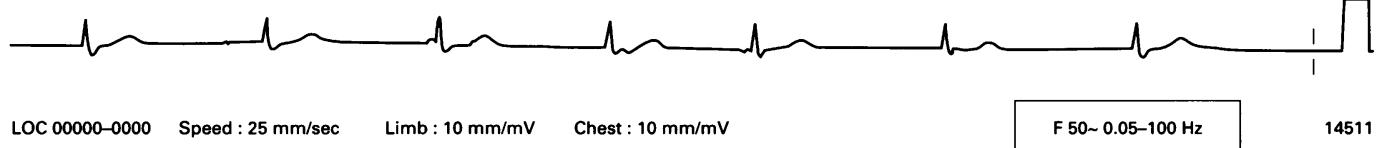

Figure 1 functional rhythm with widespread ST depression most marked in V1-V3. The $R$ waves are not dominant in these leads. The ECG findings are compatible with anterior ischaemic changes or a posterior infarction. 

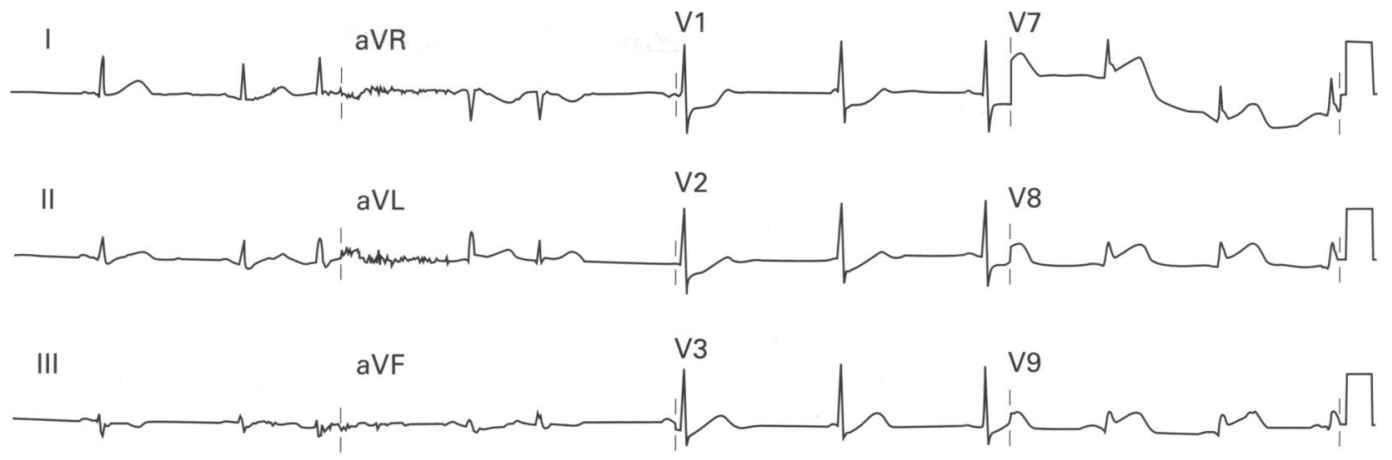

II

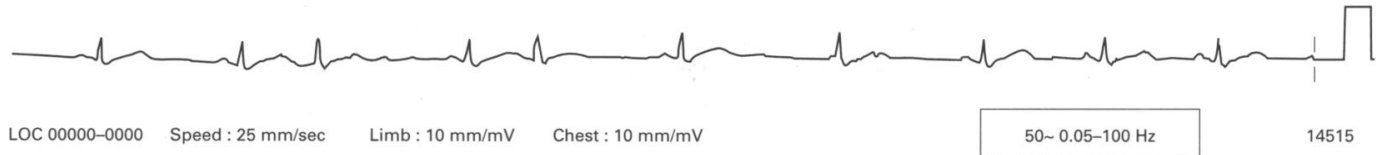

Figure 2 Demonstrates ST elevation in leads V7, V8, and V9 and confirms the presence of a posterior wall AMI. Right sided chest leads (not shown) were normal.

had felt sweaty and dizzy with near syncope. On arrival he was pale, sweaty, and hypotensive. An ECG was performed (fig 3), revealing sinus rhythm with first degree atrioventricular block, ST segment elevation in the inferior distribution, and ST segment depression in the anterior chest leads; these electrocardiographic findings were felt to be consistent with inferior wall AMI with reciprocal anterior ST segment change. The differential diagnosis of the reciprocal ST segment depression in V1-V3 included acute posterior wall infarction versus anterior wall ischaemia. Additional leads were then recorded to assist in answering this question. Figure 4, using posterior chest leads, revealed ST segment elevation in leads V8 and V9 indicating acute posterior wall infarction. Figure 5, employing right sided leads (RV1RV5), demonstrated ST segment elevation, suggestive of right ventricular myocardial infarction. Elevated cardiac enzymes confirmed the clinical diagnosis of inferoposterior AMI with right ventricle infarction.

\section{Discussion}

A more detailed description of the extent of the myocardial injury may be obtained if additional leads are used to augment the standard 12 lead ECG in selected patients. The most frequently employed additional lead ECG is the 15 lead ECG. The 15 lead ECG uses the additional lead, RV4, to investigate the right ventricle and two additional leads, V8 and V9, to image the posterior wall of the left ventricle. Neither of these areas can be properly assessed by the standard 12 lead ECG. The use of additional leads has increased the reported rate of isolated posterior AMI, suggesting that posterior myocardial infarction is often missed as the electrocardiographic features are not widely recognised. ${ }^{236}$ Failure to recognise a transmural posterior myocardial infarction on the ECG may deny these patients the opportunity to benefit from acute revascularisation treatment, such as thrombolysis or primary angioplasty. Figure 6 shows the correct placement of the additional leads RV4, V8, and V9.

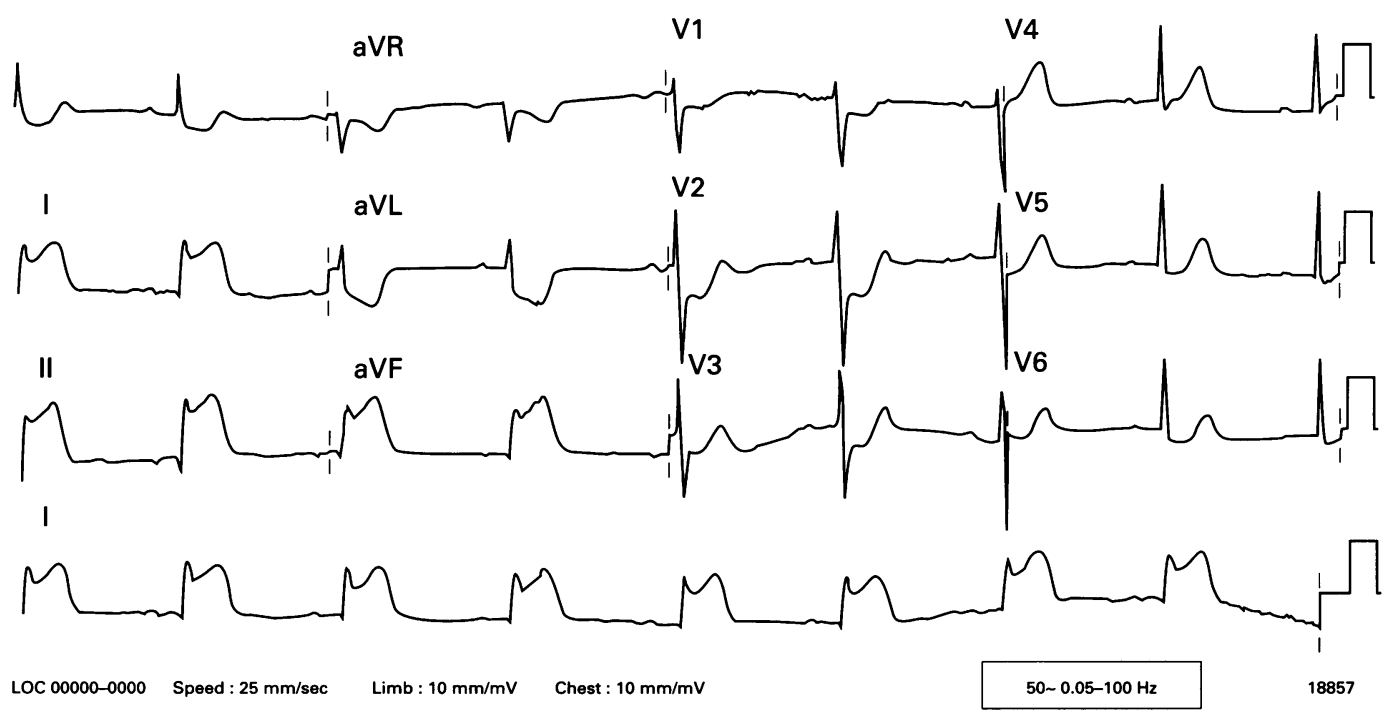

Figure $3 S R$ with first degree atrioventricular block. "Hyperacute" inferior wall ST segment elevation and widespread ST depression in the right precordial leads. 

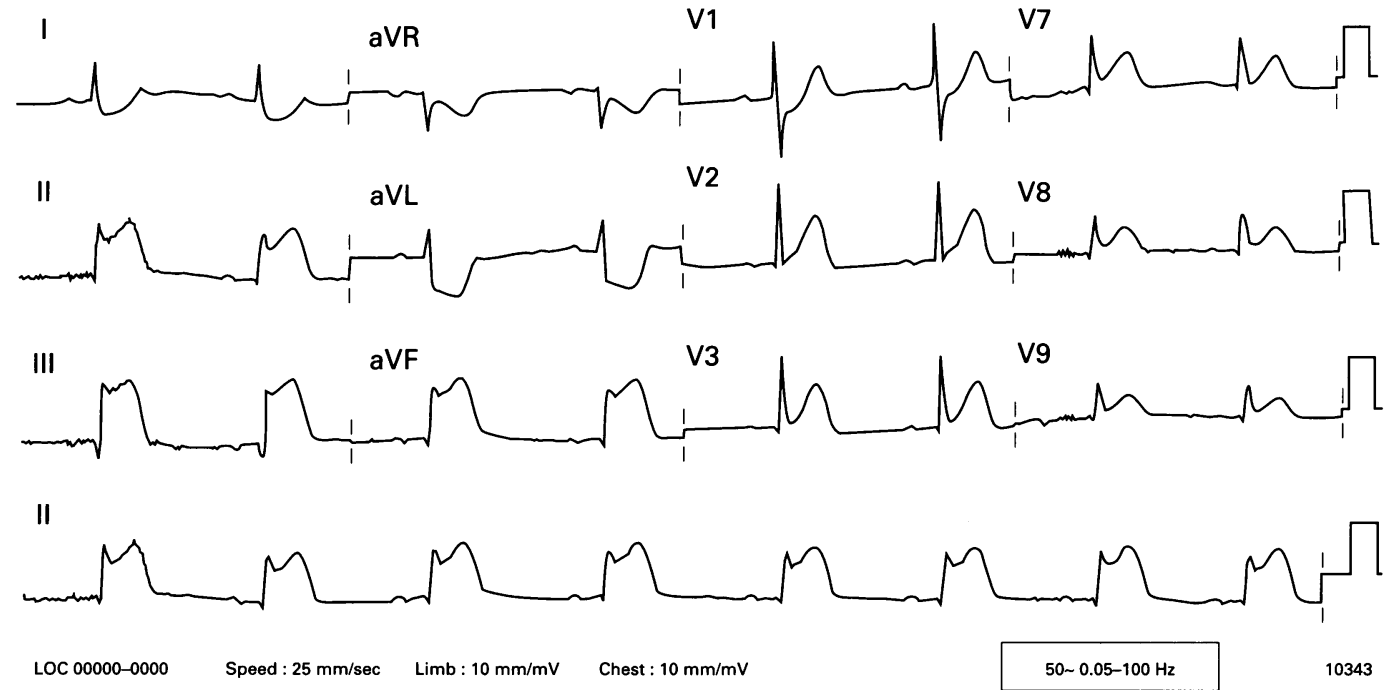

Figure 4 Posterior chest leads reveal ST segment elevation in leads V7-V9 indicating posterior wall extension of the AMI.
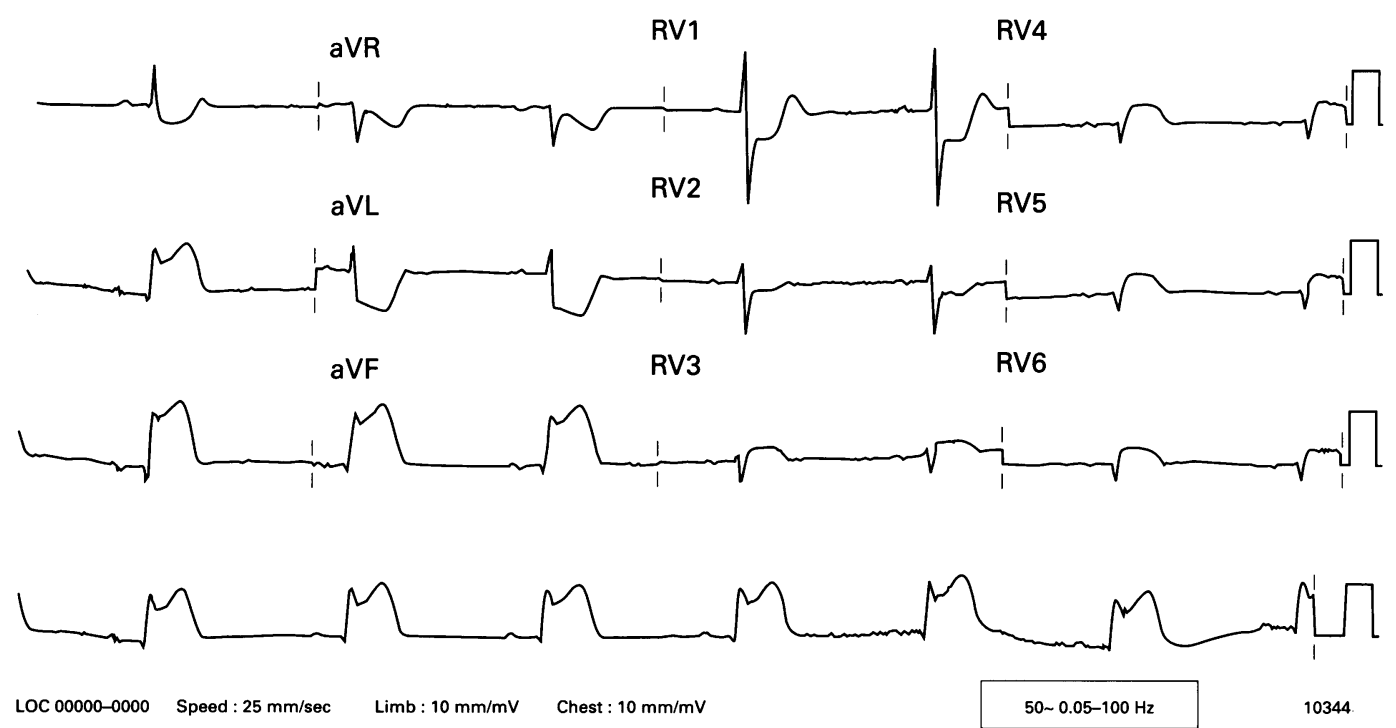

Figure 5 Right ventricular leads showing ST segment elevation confirming right ventricular infarction.

RIGHT VENTRICULAR INFARCTION

Right ventricular infarction occurs in the setting of $25 \%$ to $40 \%$ of inferior wall AMIs. A recent study documents 522 patients with inferior wall AMI with $32 \%$ of these patients experiencing right ventricular infarction ${ }^{7}$; right ventriular infarction occasionally may be an isolated phenomenon. Right ventricular in-

A

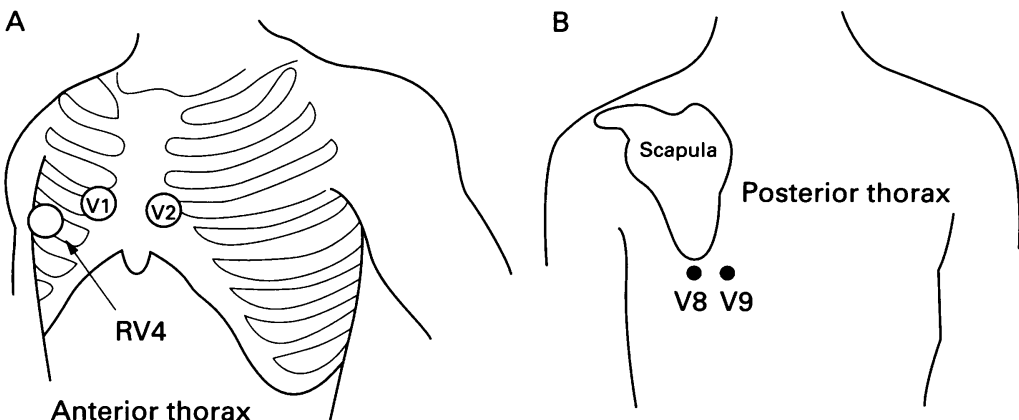

Figure 6 Correct placement of the additional leads RV4,V8, and V9 (A) Placement of lead RV4 is depicted here; this lead images the right ventricle and is placed on the right anterior thorax in an analogous position to left sided V4. (B) The posterior leads V8 and $V 9$, which directly examine the posterior wall of the left ventricle, are located on the patient's left posterior thorax in the following fashion: lead V8 at the tip of the scapula and V9 halfway between lead $V 8$ and the left border of the vertebral bodies. volvement usually occurs as a result of occlusion of the right coronary artery proximal to the right ventricular branch-with associated acute inferior wall infarction; less commonly, right ventricular infarction results from occlusion of a dominant circumflex artery in the setting of acute lateral wall myocardial infarction. In the setting of inferior AMI, the clinical findings of hypotension and raised jugular venous pressure are highly suggestive of right ventricular infarction. It has also been suggested that nitrate induced hypotension is suggestive of right ventricular infarction. It is important to diagnose right ventricular infarction, since the associated hypotension will likely respond to intravenous fluid administration whereas diuretic agents, morphine, and nitrates may further compound the situation. Patients with inferior wall AMI with coexistent right ventricular infarction have larger sized infarcts and more often experience in-hospital complications and higher cardiac mortality rates. $^{7}$

The standard 12 lead ECG findings for right ventricular infarction include ST segment 
elevation in the inferior distribution (figs 3, 5 , and 7) as well as in the right precordial chest leads, particularly lead V1-perhaps the only lead on the standard ECG which reflect changes in the right ventricle. At times, coexisting posterior wall AMI may obscure the ST segment elevation resulting from right ventricular infarction in lead V1 as seen in the

Inferior leads

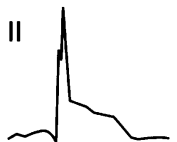

III

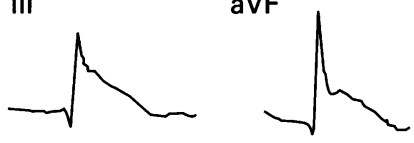

Right ventricular lead

RV4

Figure 7 Electrocardiographic complexes in a patient with inferior AMI with right ventricular involvement. The typical ST segment elevation is seen here in the inferior leads II, III, and aVF. This patient developed marked hypotension after nitroglycerine administration, suggestive of right ventricular infarct. Analysis of lead RV4 revealed ST segment elevation of approximately $1.5 \mathrm{~mm}$, diagnositc of an acute right ventricular myocardial infarction. Lead RV4 with ST segment elevation (arrow) in a patient with right ventricular myocardial infarction and coexisting inferior wall AMI. Note that the single lead RV4 is most often sufficient to make the electrocardiographic diagnosis of acute right ventricular infarction.

Right sided chest leads
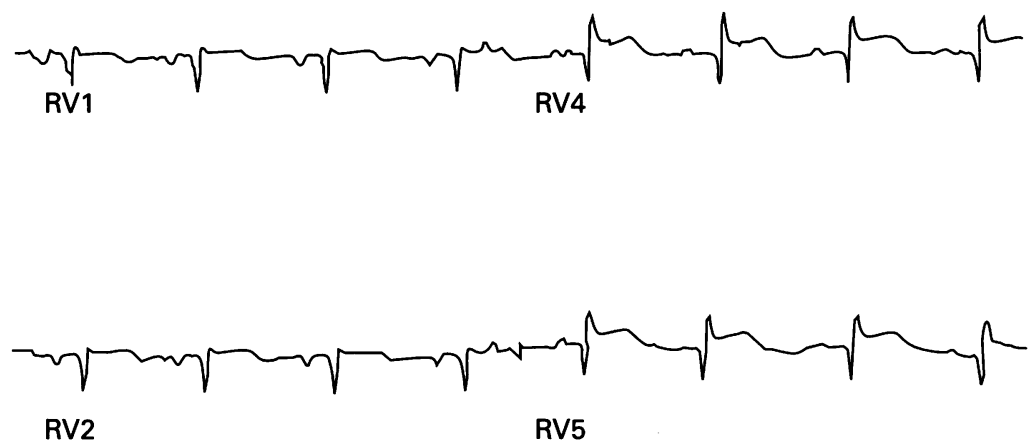

RV2

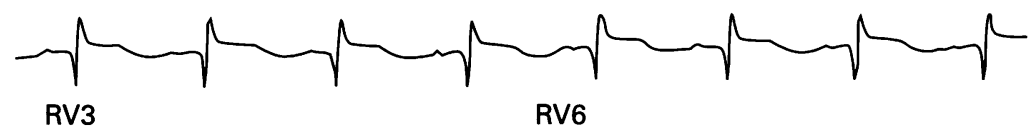

Figure 8 Entire set of right sided leads RV1-RV6 with ST segment elevation, confirming the clinical impression of right ventricular myocardial infarction in a patient with inferior wall AMI, nitrate related hypotension, and pronounced jugular venous distension.

Right precordial leads<smiles>BCCCCCC</smiles>

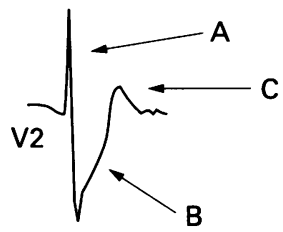

Figure 9 Electrocardiographic abnormalities suggestive of acute posterior myocardia infarction include the following (in leads V1, V2, or V3): (1) horizontal ST segment depression; (2) a tall, upright $T$ wave; (3) a tall, wide $R$ wave; and (4) an $R / S$ wave ratio greater than 1.0 (in lead V2 only). Further, the combination of horizontal ST segment depression with an upright $T$ wave increases the diagnostic accuracy of these two separate electrocardiographic findings. In this example, the right precordial leads V1 and V2 demonstrate the classic findings of acute posterior wall myocardial infarction. " $A$ " refers to the prominent $R$ wave which is unusual in the right precordial leads. " $B$ " refers to the $S T$ segment depression seen in the anterior distribution which suggests posterior wall infarction; here lead V1 shows horizontal depression. " $C$ "labels the upright T wave, when encountered in the setting of $S T$ segment depression, is strongly suggestive of acute posterior wall infarction. The posterior leads V8 and V9 demonstrate ST segment elevation-note that the degree of elevation is less pronounced than is usually associated with acute infarction.
Box 1: Electrocardiographic criteria of AMI of the posterior wall of the left ventricle using both standard 12 lead ECG and additional lead ECG

Standard 12 lead ECG

Horizontal ST segment depression*

$R$ wave with increased voltage ${ }^{\star}$

$\mathrm{R} / \mathrm{S}$ wave ratio $>1.0$ in lead $\mathrm{V} 2$ only

Prominent, upright $\mathrm{T}$ wave*

Combination of horizontal ST segment depression with upright $T$ waves ${ }^{\star}$

Coexisting acute inferior or lateral myocardial infarction

Additional lead ECG (posterior leads V8 and V9)

\section{$>1 \mathrm{~mm}$ ST segment elevation}

* Limited to leads V1, V2, and/or V3

patient with the acute inferoposterior myocardial infarction with right ventricular involvement (case 2, figs 3, 4, and 5). Recordings from leads placed on the right side of the chest are much more sensitive and specific in detecting the changes of right ventricular infarction. The right sided precordial electrodes are placed across the right side of the chest in a mirror image of the standard left sided leads and are labelled RV1-RV6; V1R-V6R is another commonly used nomenclature for this lead distribution. The clinician may use either the entire right sided leads RV1-RV6 or the single lead RV4. Lead RV4 (right fifth intercostal space mid-clavicular line), is the most useful lead for detecting ST segment elevation associated with right ventricular infarction and may be used solely in the evaluation of the possible right ventricular infarction (fig 6A). The ST segment elevation which occurs in association with right ventricular infarction is frequently quite subtle (fig 7 left), reflecting the relatively small muscle mass of the right ventricle; at other times, the ST segment elevation is quite prominent, similar in appearance to the ST segment changes seen in the standard 12 leads (fig 8). These changes are often transient, frequently resolving within 10 hours of the onset of symptoms. Right ventricular infarction may be associated with increased rates of development of atrial arrhythmia, right bundle branch block, and atrioventricular block.

POSTERIOR MYOCARDIAL INFARCTION

The term posterior myocardial infarction refers to infarction involving the posterior wall of the left ventricle, resulting from occlusion of either the right coronary artery, its posterior descending branch, or, less commonly, the circumflex artery. Posterior wall involvement occurs in $15 \%$ to $21 \%$ of all AMIs, usually in conjunction with inferior or lateral infarction. Posterior myocardial infarction can occur as an isolated phenomenon, but the incidence is probably very low. ${ }^{59}$ Patients experiencing inferior wall AMI with either right precordial ST segment depression or ST segment elevation in the posterior leads, in general, have larger sized myocardial infarctions with lower 
resultant ejection fractions and higher rates of acute cardiovascular complication and death compared to patients with inferior AMI without such changes. ${ }^{10}$ As the standard 12 lead ECG does not include posterior leads, changes associated with necrosis in this region are reflected in the anterior chest leads (figs 9
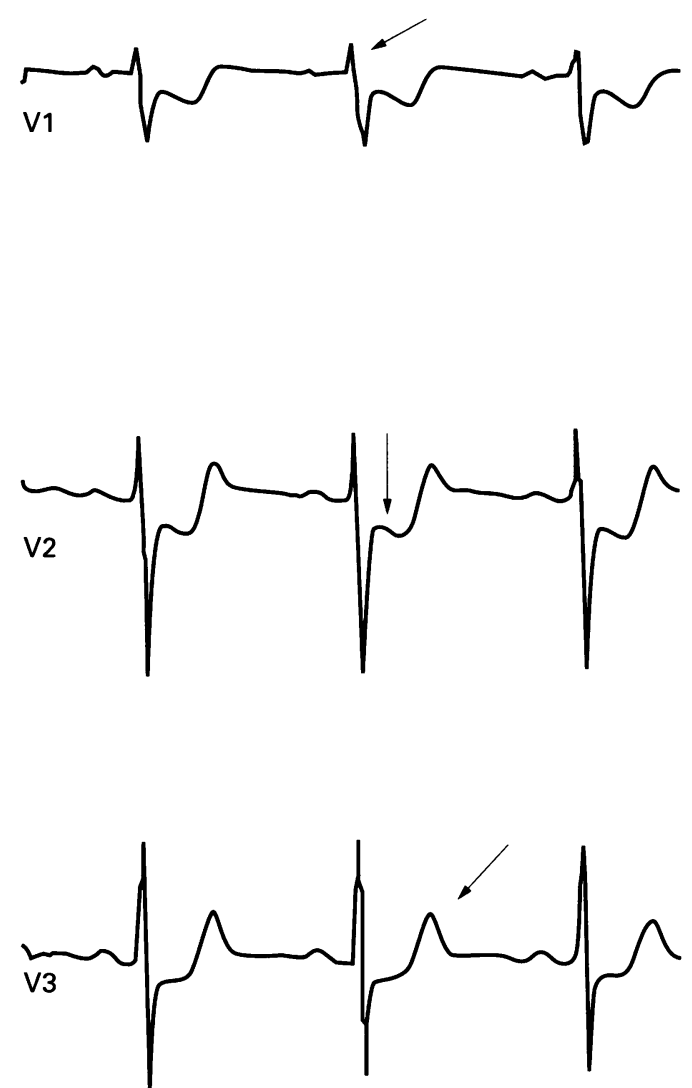

Figure 10 The right precordial leads V1-V3 show the findings associated with posterior wall $A M I$. $A$ prominent $R$ wave in lead V1 (arrow), horizontal ST segment depression (arrow in lead V2) in leads V1 through V3, and upright $T$ wave (arrow in lead V3) in leads V2 and V3 all suggest acute posterior myocardial infarction. The use of additional posterior leads (fig 12) will confirm the presence of acute posterior myocardial infarction.

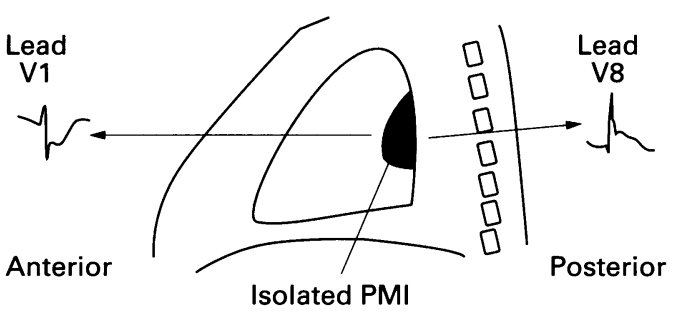

Figure 11 From the perspective of the standard 12 lead ECG, the "typical" electrocardiographic findings indicative of acute transmural myocardial infarction will be reversed. This reversal results from the fact that the endocardial surface of the posterior wall faces the anterior precordial leads (V1-V3) in the standard 12 lead ECG. In other words, $S T$ segment depression, prominent $R$ waves, and upright T waves in leads V1-V3, "when reversed", may represent $S T$ segment elevation, $Q$ waves, and $T$ wave inversions, respectively, of acute posterior myocardial infarction. If one considers the "reverse nature" of these electrocardiographic abnormalities when applied to the posterior wall, the findings assume a more recognisable, ominous meaning. In the setting of acute posterior myocardial infarction, the 12 lead ECG in the right precordial chest leads will display ST segment depression with a large $R$ wave; if viewed from the posterior perspective of the thorax (that is, the posterior thoracic leads), these same findings are "reversed" and indicate acute transmural infarction of the posterior wall of the left ventricle (PMI = posterior myocardial infarction).
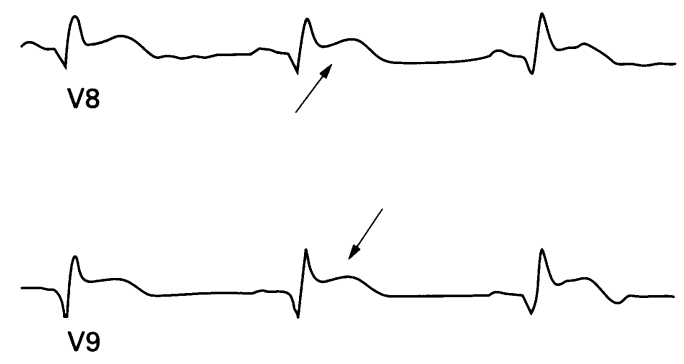

Figure 12 Posterior thorax leads (V8 and V9) corresponding to right precordial leads seen in fig 10. Note the ST segment elevation (arrows) indicative of $A M I$ of the posterior wall of the left ventricle.

and 10). ${ }^{11}$ These electrodes are opposite rather than adjacent to the site of damage and the changes seen are the reverse of what one would normally expect (fig 11). Thus the ST segment elevation that occurs with posterior myocardial infarction becomes ST segment depression in the right precordial leads, V1-V3. Abnormalities are most frequently detected in leads V1 and V2, to a lesser extent in lead V3. Electrocardiographic abnormalities (box 1) noted on the standard 12 lead ECG suggestive of acute posterior myocardial infarction include the following (in leads V1, V2, or V3): (1) horizontal ST segment depression (fig 3 and lead V1 in fig 9); (2) a tall, upright $\mathrm{T}$ wave (lead V2 in fig 9 and leads V2 and V3 in fig 10); (3) a tall, wide $\mathrm{R}$ wave (figs 3, 9, and $10^{10}$ ); and (4) an R/S wave ratio greater than or equal to 1.0 (in lead V2 only, fig 9). Further, the combination of horizontal ST segment depression with an upright $T$ wave increases the diagnostic accuracy of these two separate electrocardiographic findings. It must be remembered that a dominant $\mathrm{R}$ wave, which is in fact an evolving $Q$ wave, takes a number of hours to develop and therefore is not frequently seen on the initial ECG. ${ }^{912}$

The use of the additional posterior leads, V8 and V9, may well confirm the presence of a posterior myocardial infarction and are felt to be superior to the findings noted in leads V1 through to V3. The V8 electrode is placed at the tip of the left scapula and V9 midway between V8 and the spinous processes of the dorsal spine (fig 6B). Posterior leads should be recorded in any patient with ST segment depression in the right precordial chest leads or with ST segment elevation in the inferior or lateral leads. When ST segment depression is present in V1-V3, the use of posterior chest leads may help differentiate between patients with anterior wall ischaemia and those with posterior myocardial infarction. Boden et al demonstrated that approximately $50 \%$ of such patients may have posterior wall damage. ${ }^{5}$ The degree of ST segment elevation in the posterior leads (fig 12) may not be pronounced-not unlike the magnitude of ST segment change in the right ventricular infarction. Such "less pronounced" posterior lead ST segment elevation, however, occurs for a different reason. The posterior leads are relatively distant from the posterior wall of the left ventricle in contrast to the standard precordial leads V1-V6. 


\section{Conclusion}

The use of the 15 lead ECG may help to define the full extent of the myocardial injury in patients with AMI. The use of additional leads may confirm the diagnosis of right ventricular infarction in hypotensive patients presenting with an inferior AMI. In patients with ST depression in leads V1-V3, the use of the additional leads V8 and V9 may help to distinguish between a posterior myocardial infarction, anterior wall ischaemia, and reciprocal changes. The 15 lead ECG is recommended in any patient presenting with an inferior myocardial infarction, any infarction involving the lateral wall of the left ventricle or any patient with ST depression in leads V1-V3. The 15 lead ECG is not routinely recorded in accident and emergency departments at present. It is used much more frequently in emergency departments in hospitals in the United States. Indeed the National Heart Attack Alert programme working groups have suggested that the use of 15 leads rather than the standard 12 may assist the doctor in certain circumstances. ${ }^{12}$ We advocate the widespread use of the 15 lead ECG in selected patients as it will help to inform not only the inexperienced as well as demonstrating the full extent of the AMI.

Conflict of intertest: none.

Funding: none.

1 Rude RE, Poole WK, Muller JE, et al. Electrocardiographic and clinical criteria for recognition of acute myocardial infarction based on analysis of 3697 patients. Am $\mathcal{f}$ Cardiol 1983;52:936-42.
2 Pollack M, Thomason G, Williams M, et al. Emergency department diagnosis of acute posterior-wall myocardial infarction using left posterior chest leads. Acad Emerg Med 1997;34:399.

3 Melendez LJ, Jones DT, Salcedo JR. Usefulness of three additional electrocardiographic chest leads (V7, V8 and V9) in the diagnosis of acute myocardial infarction. Can V9) in the diagnosis of acute
Med Assoc $\mathcal{F} 1978 ; 119: 745-8$.

4 Rich MW, Imburgia M, King TR, et al. Electrocardiographic diagnosis of remote posterior wall myocardial infarction using unipolar posterior lead V9. Chest 1989;96: 489-93.

5 Boden WE, Kleiger RE, Gibson RS, et al. Electrocardiographic evolution of posterior acute myocardial infarction: importance of early precordial ST-segment depression. $\mathrm{Am}$ importance of early precor

6 Perloff JK. The recognition of strictly posterior myocardial infraction by conventional scalar electrocardiography. Circulation 1964;30:706-18.

7 Zeymer U, Neuhaus KL, Wegscheider K, et al. Effects of thrombolytic therapy in acute myocardial infarction with or without right ventricular involvement. F $\mathrm{Am}$ Coll Cardiol 1998;32:876-81.

8 Brady WJ. Chang N, Hwang V, et al. The 15-lead ECG in emergency department chest pain patients: comparison to the 12-lead ECG. Presented at the International Emergency Medicine Conference, Vancouver, British Columbia, Canada, March 1998.

9 Goldberger AL. Myocardial infarction: electrocardiographic differential diagnosis. 4th Ed. St Louis, MO: Mosby, 1991.

10 Matetzky S, Freimark D, Chouraqui P, et al. Significance of ST segment elevations in posterior chest leads (V7 to V9) in patients with acute inferior myocardial infarction: appliin patients with acute inferior myocardial infarction: appli-
cation for thrombolytic therapy. $₹ \mathrm{Am}$ Coll Cardiol 1998;31: 506-11.

11 Conover MB. Understanding electrocardiography: arrhythmias and the 12-lead ECG. 6th Ed. St Louis, MO: Mosby, 1992.

12 Aufderheide TP, Brady WJ. Electrocardiography in the patient in myocardial ischaemic or infarction. In: Gibler WB, Aufderheide TP, eds. Emergency cardiac care. St Louis, MO: Mosby, 1994.

13 Selker HP, Zalenski RJ, Antman EM, et al. An evaluation of the technologies for identifying acute cardiac ischameic in the emergency department: a report from the National Heart Attack Alert Program Working Group. Nonstandard ECG leads and body surface mapping. Ann Emerg Med 1997;29:28-33. 